\title{
A New X-bar Control Chart for Multiple Dependent State Sampling Using Neutrosophic Exponentially Weighted Moving Average Statistics with Application to Monitoring Road Accidents and Road Injuries
}

\author{
Nasrullah Khan ${ }^{1}$. Liaquat Ahmad² G. Srinivasa $\operatorname{Rao}^{3} \cdot$ Muhammad Aslam $^{4}$ D . Ali Hussein AL-Marshadi ${ }^{4}$
}

Received: 9 July 2021 / Accepted: 30 September 2021

(c) The Author(s) 2021

\begin{abstract}
In this article, an efficient mean chart for symmetric data have been presented for multiple dependent state (MDS) sampling using neutrosophic exponentially weighted moving average (NEWMA) statistics. The existing neutrosophic exponentially weighted moving average charts are not capable of seizure the unusual changes threatened to the manufacturing processes. The control chart coefficients have been estimated using the symmetry property of the Gaussian distribution for the uncertain environment. The neutrosophic Monte Carlo simulation methodology has been developed to check the efficiency and performance of the proposed chart by calculating the neutrosophic average run lengths and neutrosophic standard deviations. The proposed chart has been compared with the counterpart charts for confirmation of the proposed technique and found to be a robust chart.
\end{abstract}

Keywords MDS sampling · Neutrosophic statistic · EWMA chart · Average run length · Monte Carlo simulation

\section{Introduction}

Control charts have been used extensively in a variety of areas like manufacturing processes [1-3], goods and services providing companies [4], health care enterprises [5],

Muhammad Aslam

aslam_ravian@hotmail.com

Nasrullah Khan

nas_shan1@hotmail.com

Liaquat Ahmad

liaquatahmad@uvas.edu.pk

G. Srinivasa Rao

gaddesrao@gmail.com

Ali Hussein AL-Marshadi

aalmarshadi@kau.edu.sa

1 Department of Statistics, University of Veterinary and Animal Sciences, Jhang Campus, Lahore, Pakistan

2 Department of Statistics and Computer Science, University of Veterinary and Animal Sciences, Lahore, Pakistan

3 Department of Mathematics and Statistics, The University of Dodoma, PO. Box: 259, Dodoma, Tanzania

4 Department of Statistics, Faculty of Science, King Abdulaziz University, Jeddah 21551, Saudi Arabia nuclear engineering [6, 7], Software industry [8, 9], education [10], analytical laboratories [11, 12], etc. since its inception floated by Walter A. Shewhart during 1920s. The Statistical Process Control (SPC) experts are struggling hard to devise a robust control chart technique for the efficient monitoring of the process but in vain to launch a universal control chart methodology. Several modifications by SPC researchers have been proposed by mixing and combining the design structures of monitoring techniques. The multiple dependent state (MDS) sampling technique combined with a fixed deferred state sampling scheme for the correct decision of lot sentencing was promoted by Wortham and Baker [13]. The MDS sampling in combination with the double control limits can be used for the efficient monitoring of the production process [14]. The combination of exponentially distributed quality characteristics with the MDS has been shown as an efficient monitoring technique for the mean of the production process [15]. Further literature on proposed approaches for quick detection of unusual changes can be consulted [16-20].

The fuzzy approach is very common in the control chart literature due to its efficient capability of handling vague data [21]. Ambiguous and unclear data are well represented by the fuzzy logic which provides a systemic base by using algorithms of defuzzification methods [21]. The fuzzy approach 
provides the perfect means of dealing with human subjectivity by modeling uncertainty which is neither stochastic nor random. The fuzzy control charts are inevitable to deal with uncertain, vague, incomplete or data collected on human subjectivity. Several procedures have been proposed to develop fuzzy control charts since the inception of fuzzy set theory by Zadeh [22]. Fuzzy set theory can be used to develop the control chart for quality assurance of the industrial product when the observations are collected from linguistic terms [23]. A methodology for developing a quantitative control chart for nonprecise observations was given by Gülbay and Kahraman [24]. A hybrid fuzzy adaptive control chart was developed to enhance the performance of the Shewhart control chart using fuzzified sensitivity criteria by Zarandi et al. [25]. The X-bar chart using the fuzzy theorem for non-crispy data using the triangular fuzzy membership function was developed by Panthong and Pongpullponsak [26]. An attribute sampling plan under the multiple dependent state sampling for the fuzzy environment was designed by Afshari and Sadeghpour Gildeh [27]. Control charts under fuzzy theory for the univariate case and multivariate case were developed by Fernández [28]. Fuzzy logic is the special case of neutrosophic logic.

Neutrosophic statistics has attracted the attention of several SPC experts during the last few years due to its nice properties of analyzing the vague, incomplete, imprecise, unclear and uncrispy data from numerous practical, realworld and everyday circumstances. Neutrosophic logic was introduced by Smarandache [29]. The engineering rock mass using the joint roughness coefficient under the neutrosophic statistics for scale effect and anisotropy environment were analyzed by Chen et al. [30]. Sampling plan under the neutrosophic process loss consideration was developed by Aslam [31]. Sampling plans using regression estimators for the neutrosophic statistics through the neutrosophic optimization solution were presented by Aslam and AL-Marshadi [32]. Control charts for failure-censored reliability tests under the neutrosophic environment were presented by Aslam et al. [33]. Dispersion control charts using the neutrosophic statistical interval method were presented by Aslam et al. [34]. A control chart based upon gamma-distributed quality characteristic under the neutrosophic statistics was developed by Aslam et al.[35]. Shewhart S-control chart for process variability monitoring using the neutrosophic statistics was presented by Khan et al. [36]. A robust singlevalued neutrosophic soft aggregation operator in multi-criteria decision-making was developed by Jana and Pal [37]. Trapezoidal neutrosophic aggregation operators and their application to the multi-attribute decision-making process are given by Jana et al. [38]. Multi-criteria decision making approach based on SVTrN Dombi aggregation functions proposed by Jana et al. [39]. Multi-criteria decision making process based on some single-valued neutrosophic Dombi power aggregation operators was developed by Jana and Pal
[40]. Multiple-attribute decision making problems based on SVTNH methods were studied by Jana et al. [41]. More literature on neutrosophic statistics are suggested as [42-58].

The performance of the newly developed chart can be evaluated by estimating the average run lengths (ARL) which is defined as the average number of samples falling in-control limits until it indicates the out-of-control process [59]. The existing classical or neutrosophic exponentially weighted moving average charts are not accomplished to capture the extraordinary changes vulnerable to the manufacturing processes. The EWMA chart under neutrosophic statistics using single sampling was developed by Aslam et al. [60]. By exploring the literature and according to the best of our knowledge, there is no work on EWMA chart under neutrosophic statistics using MDS sampling. The present control chart has been using the symmetry property of the Gaussian distribution for the uncertain environment. We will design the proposed control chart and compare the efficiency with the existing chart. We expect that the proposed chart will be efficient than the existing chart in detecting the shift earlier. The rest of the paper is organized as follows: the methodology of the proposed chart is given in Sect. 2. Control chart based on multiple dependent state sampling is given in Sect. 3. The simulation study for proposed neutrosophic control chart is addressed in Sect. 4. A comparison of the proposed chart has been given in Sect. 5. In Sect. 6, the application of the proposed methodology is discussed. Conclusion and recommendations have been stated in Sect. 7.

\section{Methodology of the Proposed Chart}

This section deals with proposing the NEWMA statistics. The neutrosophic random variable (NRV) is denoted as $Y_{j N} \in\left[Y_{L}, Y_{U}\right] ; j=1,2, \ldots, n_{N}$, where $n_{N}$ is the neutrosophic sample size (NSS). Assume that the neutrosophic sample mean (NSM) and sample variance are defined as $\bar{Y}_{N} \in\left[\frac{\sum_{j=1}^{n_{L}} Y_{j}}{n_{L}}, \frac{\sum_{j=1}^{n_{U}} Y_{j}}{n_{U}}\right] ; \bar{Y}_{N} \in\left[\bar{Y}_{L}, \bar{Y}_{U}\right]$ and $S_{N}^{2}=\sum_{j=1}^{n_{N}}$ $\left(Y_{N}-\bar{Y}_{N}\right)^{2} /\left(n_{N}-1\right) ; S_{N}^{2} \in\left[S_{L}^{2}, S_{L}^{2}\right]$. According to Smarandache [29] and Aslam [31], the NSM comes from the neutrosophic normal distribution (NND) with a neutrosophic population mean and variance are respectively $\mu_{N}$ and $\sigma_{N}^{2} / n_{N}$, where $\mu_{N}=\sum_{j=1}^{N_{N}} Y_{N} / N_{N} ; \mu_{N} \in\left[\mu_{L}, \mu_{U}\right]$ and $\sigma_{N}^{2}=\sum_{j=1}^{n_{N}}\left(Y_{N}-\mu_{N}\right)^{2} /\left(N_{N}-1\right) ; \sigma_{N}^{2} \in\left[\sigma_{L}^{2}, \sigma_{U}^{2}\right]$. Using foresaid acquaintance the NEWMA statistics is given below:

$$
\begin{aligned}
\operatorname{EWMA}_{N, j} & =\lambda_{N} \bar{Y}_{N}+\left(1-\lambda_{N}\right) \mathrm{EWMA}_{N, j-1} ; \\
\mathrm{EWMA}_{N, j} & \in\left[\mathrm{EWMA}_{L, j}, \mathrm{EWMA}_{U, j}\right]
\end{aligned}
$$


where $\lambda_{N}$ is neutrosophic smoothing constant, $\lambda_{N} \in\left[\lambda_{L}, \lambda_{U}\right]$ and the range of $\lambda_{N}$ is $[0,0] \leq \lambda_{N} \leq[1,1]$. Note here that $\bar{X}_{N} \in\left\{\bar{X}_{L}, \bar{X}_{U}\right\}$ are assumed to be independent random variables with neutrosophic variance $\sigma_{N}^{2} / n_{N}$; $\sigma_{N}^{2} / n_{N} \in\left[\sigma_{L}^{2} / n_{N}, \sigma_{L}^{2} / n_{N}\right]$ and known neutrosophic population variance, as shown in [61]. Montgomery [62] suggested that the smoothing constant should ranges from 0.05 to 0.25 . The EWMA $_{N, j}$ comes from the NND with neutrosophic mean as $\mu_{N} \in\left[\mu_{L}, \mu_{U}\right]$ and neutrosophic standard deviation as $\frac{\sigma_{N}}{\sqrt{n_{N}}} \sqrt{\frac{\lambda_{N}}{2-\lambda_{N}}}$.

\section{The Proposed NEWMA X-Bar Control Chart Based on Multiple Dependent State Sampling}

The NEWMA X-bar control chart using the MDS sampling plan under the NS is outlined in the below steps:

1. Drawn an NRV of size $n_{N} \in\left[n_{L}, n_{U}\right]$ and determine EWMA $_{N, j}$ statistics given in Eq. (1).

2. Assert the process is stated as in-control when $\operatorname{LCL}_{N} \leq \mathrm{EWMA}_{N, i} \leq \mathrm{UCL}_{N}$. Admit the process as out-of-control when $\mathrm{EWMA}_{N, j} \geq \mathrm{UCL}_{N}$ or $\mathrm{EWMA}_{N, j} \leq \mathrm{LCL1}_{N}$. If not move to step 3 . Note here that $\operatorname{LCL}_{N} \in\left[\mathrm{LCL}_{L}, \mathrm{LCL}_{U}\right]$ and $\mathrm{UCL}_{N} \in\left[\mathrm{UCL}_{L}, \mathrm{UCL}_{U}\right]$ are outer neutrosophic limits; $\operatorname{LCL} 2_{N} \in\left[\mathrm{LCL}_{L}, \mathrm{LCL}_{U}\right]$ and $\mathrm{UCL} 2_{N} \in\left[\mathrm{UCL} 2_{L}, \mathrm{UCL} 2_{U}\right]$ are the inner neutrosophic limits respectively.

3. Pronounce the process is said to be in-control if $m_{N}$ preceding subgroups have been affirming the process to be in-control.

$$
\begin{aligned}
\mathrm{UCL}_{N}= & \mu_{N}+k 2_{N} \frac{\sigma_{N}}{\sqrt{n_{N}}} \sqrt{\frac{\lambda_{N}}{2-\lambda_{N}}} ; \mathrm{UCL} 2_{N} \in \\
& {\left[U C L 2_{L}, U C L 2_{U}\{\{\}\}\right] ; k 2_{N} \in } \\
& {\left[k 2_{L}, k 2_{U}\right], \mu_{N} \in\left[\mu_{L}, \mu_{U}\right] }
\end{aligned}
$$

Where $k 1_{N} \in\left[k 1_{L}, k 1_{U}\right]$ and $k 2_{N} \in\left[k 2_{L}, k 2_{U}\right]$ are the neutrosophic control limits coefficients. The developed control chart becomes an existing NS control chart proposed by [60] when $k 1_{N}=k 2_{N}=k_{N}, m_{N}=1$ and developed control chart becomes a chart developed by [52] when $\lambda_{N} \in\{1,1\}$. When $k 1_{N}=k 2_{N}=k_{N}=k, m_{N}=m=1$ the developed control chart becomes the classical Shewhart chart. Let $\mu_{0 N} \in\left[\mu_{0 L}, \mu_{0 U}\right]$ is a target mean value for the process. The probability of the process under the NS is an in-control state for the proposed control chart is given below:

$$
\begin{aligned}
P_{i n N}^{0}= & P\left(\mathrm{LCL}_{N} \leq \mathrm{EWMA}_{N, j} \leq \mathrm{UCL}_{N} \mid \mu_{0 N}\right) \\
& +\left\{\begin{array}{c}
P\left(\mathrm{LCL}_{N} \leq \mathrm{EWMA}_{N, j} \leq \mathrm{LCL} 2_{N} \mid \mu_{0 N}\right)+ \\
P\left(\mathrm{UCL}_{N} \leq \mathrm{EWMA}_{N, j} \leq \mathrm{UCL}_{N} \mid \mu_{0 N}\right)
\end{array}\right\} \\
& \left\{P\left(\mathrm{LCL}_{N} \leq \mathrm{EWMA}_{N, j} \leq \mathrm{UCL}_{N} \mid \mu_{0 N}\right)\right\}^{m_{N}}
\end{aligned}
$$

The neutrosophic average run length (NARL) for the developed control chart is as follows:

$\mathrm{ARL}_{0 N}=\frac{1}{1-P_{i n N}^{0}} ; \mathrm{ARL}_{0 N} \in\left[\mathrm{ARL}_{0 L}, \mathrm{ARL}_{0 U}\right]$

The neutrosophic probability of an in-control state when the process has shifted from $\mu_{0 N}$ to a new target at $\mu_{1 N}=\mu_{0 N}+\delta \sigma_{N} ; \mu_{1 N} \in\left[\mu_{1 L}, \mu_{1 U}\right]$, where $\delta$ is the shift constant is as follows:

Thus the proposed neutrosophic control limits are given below:

$$
\begin{aligned}
& \operatorname{LCL}_{N}=\mu_{N}-k 1_{N} \frac{\sigma_{N}}{\sqrt{n_{N}}} \sqrt{\frac{\lambda_{N}}{2-\lambda_{N}}} ; \operatorname{LCL}_{N} \in\left[\operatorname{LCL}_{L}, \operatorname{LCL}_{U}\right] ; k 1_{N} \in\left[k 1_{L,}, k 1_{U}\right] \\
& \mathrm{UCL}_{N}=\mu_{N}+k 1_{N} \frac{\sigma_{N}}{\sqrt{n_{N}}} \sqrt{\frac{\lambda_{N}}{2-\lambda_{N}}} ; \mathrm{UCL}_{N} \in\left[U C L 1_{L}, U C L 1_{U}\right] ; k 1_{N} \in\left[k 1_{L}, k 1_{U}\right] ; \mu_{\mathrm{N}} \in\left[\mu_{\mathrm{L}}, \mu_{\mathrm{U}}\right] \\
& \operatorname{LCL}_{N}=\mu_{N}-k 2_{N} \frac{\sigma_{N}}{\sqrt{n_{N}}} \sqrt{\frac{\lambda_{N}}{2-\lambda_{N}}} ; \operatorname{LCL} 2_{N} \in\left[\operatorname{LCL} 2_{L}, \operatorname{LCL}_{U}\right] ; k 2_{N} \in\left[k 2_{L,}, k 2_{U}\right], \\
& \mu_{\mathrm{N}} \in\left[\mu_{\mathrm{L}}, \mu_{\mathrm{U}}\right]
\end{aligned}
$$




$$
\begin{aligned}
P_{i n N}^{1}= & P\left(\operatorname{LCL}_{N} \leq \mathrm{EWMA}_{N, j} \leq \mathrm{UCL} 2_{N} \mid \mu_{1 N}\right) \\
& +\left\{\begin{array}{c}
P\left(L C L 1_{N} \leq E W M A_{N, j} \leq L C L 2_{N} \mid \mu_{1 N}\right)+ \\
P\left(U C L 2_{N} \leq E W M A_{N, j} \leq U C L 1_{N} \mid \mu_{1 N}\right)
\end{array}\right\} \\
& \left\{P\left(\operatorname{LCL} 2_{N} \leq \mathrm{EWMA}_{N j} \leq \mathrm{UCL}_{N} \mid \mu_{1 N}\right)\right\}^{m_{N}}
\end{aligned}
$$

The NARL at $\mu_{1 N} \in\left[\mu_{1 L}, \mu_{1 U}\right]$ is given below:

$$
\mathrm{ARL}_{1 N}=\frac{1}{1-P_{i n N}^{1}} ; \mathrm{ARL}_{1 N} \in\left[\mathrm{ARL}_{1 L}, \mathrm{ARL}_{1 U}\right]
$$

\section{The Simulation Study for Proposed Neutrosophic Control Chart}

The neutrosophic average run length (NARL) and the neutrosophic standard deviation of run length (NSDRL) are usually used to investigate the accomplishment of the control charts. The neutrosophic Monte Carlo simulation is used to estimate neutrosophic control limits constants $k 1_{N} \in\left[k 1_{L}, k 1_{U}\right]$ and $k 2_{N} \in\left[k 2_{L}, k 2_{U}\right]$ and hence, $\mathrm{ARL}_{0 N} \in\left[\mathrm{ARL}_{0 L}, \mathrm{ARL}_{0 U}\right]$. To compute the in-control state neutrosophic control limits constants $k 1_{N} \in\left[k 1_{L}, k 1_{U}\right]$ and $k 2_{N} \in\left[k 2_{L}, k 2_{U}\right]$ generate a neutrosophic random sample of size $n_{N} \in\left[n_{L}, n_{U}\right]$ from standard normal distribution. Compute the chart statistics EWMA $_{N, j}$, determine the in-control average run length $\mathrm{ARL}_{0 N} \in\left[\mathrm{ARL}_{0 L}, \mathrm{ARL}_{0 U}\right]$ and chart constants $k 1_{N}$ and $k 2_{N}$. The chart constants are determined such that $A R L_{0 N} \approx r_{0 N}$, here $r_{0 N}$ could be planned value of $\mathrm{ARL}_{0 N} \in\left[\mathrm{ARL}_{0 L}, \mathrm{ARL}_{0 U}\right]$. To obtain $\mathrm{ARL}_{0 N} \in\left[\mathrm{ARL}_{0 L}, \mathrm{ARL}_{0 U}\right]$ and chart constants $k 1_{N}$ and $k 2_{N}$ we have performed 10,000 simulation runs. Once after obtaining the chart constants, $\mathrm{ARL}_{0 N} \in\left\{\mathrm{ARL}_{0 L}, \mathrm{ARL}_{0 U}\right\}$ and NSDRL value based on 10,000 simulation runs, we need to study the out-of-control performance of the proposed control chart.

To obtain the out-of-control average run length $\mathrm{ARL}_{1 N} \in\left\{\mathrm{ARL}_{1 L}, \mathrm{ARL}_{1 U}\right\}$, shifted the process from $\mu_{0 N}$ to a new target at $\mu_{1 N}=\mu_{0 N}+\delta \sigma_{N} ; \mu_{1 N} \in\left[\mu_{1 L}, \mu_{1 U}\right]$, where $\delta$ is the shift constant. Using the chart constants obtained from the in-control process, now generate random numbers of size $n_{N}$ from normal distribution with mean $\mu_{1 N}$ and variance $\sigma_{N}^{2}$ and obtain $\mathrm{ARL}_{1 N} \in\left\{\mathrm{ARL}_{1 L}, \mathrm{ARL}_{1 U}\right\}$ for various shift values of $\delta$. Repeat the entire procedure for 10,000 iterations and compute the $\mathrm{ARL}_{1 N} \in\left\{\mathrm{ARL}_{1 L}, \mathrm{ARL}_{1 U}\right\}$ values and NSDARL values. The results are displayed in Tables 1, 2, 3 and 4 for various shift values of $\delta, n_{N}, m_{N}, \lambda_{N}$ and $A R L_{0 N}$. The values of NARL when $n_{N}=[3,5] ; m_{N}=[2,4] ; \lambda_{N}=[0.08,0.12]$ are displayed in Table 1 . The values of NARL w h e n $\quad n_{N}=[3,5] ; m_{N}=[2,4] \operatorname{and} \lambda_{N}=[0.18,0.22]$ are given in Table 2 . The values of NARL when $n_{N}=[3,5] ; m_{N}=[2,4] ; \lambda_{N}=[0.28,0.32]$ are depicted in Table 3 . The values of NARL when $n_{N} \in[5,7]$, $n_{N} \in[8,10], m_{N}=[2,4]$ and $\lambda_{N} \in[0.08,0.12]$ are shown in Table 4. The importance of the proposed control chart can found in Tables 1, 2, 3 and 4, the results show that the NARL and NSDRL are increased when $\lambda_{N} \in\left[\lambda_{L}, \lambda_{U}\right]$ values increase. It is significant to note that as shift parameter increases then there is a decreasing tendency in NARL and NSDRL values. Furthermore, from Table 4 it is interesting to note that the indeterminacy interval in NARL and NSDRL are decreases as $n_{N} \in\left[n_{L}, n_{U}\right]$ increases from $n_{N} \in[5,7]$ to $n_{N} \in[8,10]$. Hence we conclude that the indeterminacy interval in NARL and NSDRL are decreased as NSS increases.
Table 1 NARL and NSDRL

\begin{tabular}{|c|c|c|c|c|}
\hline \multirow{4}{*}{$\begin{array}{l}k 1_{N} \\
k 2_{N} \\
\delta\end{array}$} & \multirow{2}{*}{\multicolumn{2}{|c|}{$\frac{r_{0}=300}{[2.71534,3.33017]}$}} & \multirow{2}{*}{\multicolumn{2}{|c|}{$\frac{r_{0}=370}{[2.70613,2.98759]}$}} \\
\hline & & & & \\
\hline & \multicolumn{2}{|c|}{$[2.36204,2.42202]$} & \multicolumn{2}{|c|}{$[2.49637,2.52893]$} \\
\hline & NARL & NSDRL & NARL & NSDRL \\
\hline 0.00 & {$[302.88,299.88]$} & [290.18,293.27] & {$[378.95,382.23]$} & {$[364.49,375.83]$} \\
\hline 0.05 & {$[210.81,197.3]$} & {$[203.91,190.27]$} & {$[254.12,235.41]$} & {$[251.11,233.44]$} \\
\hline 0.10 & {$[111.12,90.02]$} & {$[109.98,86.06]$} & {$[128.67,108.11]$} & {$[125.02,105.62]$} \\
\hline 0.15 & {$[56.62,45.30]$} & {$[58.06,44.43]$} & {$[66.95,50.16]$} & {$[66.19,48.64]$} \\
\hline 0.20 & {$[29.90,24.31]$} & {$[32.85,23.77]$} & {$[35.71,27.13]$} & {$[39.41,26.94]$} \\
\hline 0.25 & {$[15.19,13.89]$} & {$[19.01,13.54]$} & {$[17.75,16.64]$} & {$[21.19,15.5]$} \\
\hline 0.30 & {$[7.54,8.69]$} & {$[10.0,7.66]$} & {$[8.94,9.40]$} & {$[11.8,8.24]$} \\
\hline 0.40 & {$[3.30,5.41]$} & {$[1.99,1.90]$} & {$[3.50,5.54]$} & {$[2.60,2.17]$} \\
\hline 0.50 & {$[3.01,5.02]$} & {$[0.30,0.32]$} & {$[3.01,5.03]$} & {$[0.21,0.46]$} \\
\hline 0.60 & {$[3,5]$} & {$[0,0.03]$} & {$[3,5]$} & {$[0,0.03]$} \\
\hline
\end{tabular}
values of under MDS sampling when $n_{N}=[3,5] ; m_{N}=[2,4]$; $\lambda_{N}=[0.08,0.12]$ 
Table 2 The values of NARL and NSDRL under MDS sampling when $n_{N}=[3,5] ; m_{N}=[2,4]$; $\lambda_{N}=[0.18,0.22]$

\begin{tabular}{|c|c|c|c|c|}
\hline \multirow{4}{*}{$\begin{array}{l}k 1_{N} \\
k 2_{N} \\
\delta\end{array}$} & \multicolumn{2}{|l|}{$r_{0}=300$} & \multicolumn{2}{|l|}{$r_{0}=370$} \\
\hline & \multicolumn{2}{|c|}{$[3.13326,3.35852]$} & \multicolumn{2}{|c|}{$[3.26462,3.21227]$} \\
\hline & \multicolumn{2}{|c|}{$[2.43913,2.44254]$} & \multicolumn{2}{|c|}{$[2.51606,2.54134]$} \\
\hline & NARL & NSDRL & NARL & NSDRL \\
\hline 0.00 & {$[300.96,300.34]$} & [298.74,293.57] & {$[377.63,373.75]$} & {$[369.47,365.60]$} \\
\hline 0.05 & {$[242.82,217.48]$} & {$[236.01,212.83]$} & {$[299.04,266.92]$} & {$[291.09,261.83]$} \\
\hline 0.10 & {$[150.9,115.57]$} & {$[148.27,111.13]$} & {$[179.62,142.13]$} & {$[176.31,138.23]$} \\
\hline 0.15 & {$[88.84,63.00]$} & {$[84.90,59.41]$} & {$[102.83,74.00]$} & {$[99.50,72.11]$} \\
\hline 0.20 & {$[53.85,36.27]$} & {$[52.99,33.55]$} & {$[62.25,42.16]$} & {$[60.66,39.42]$} \\
\hline 0.25 & {$[34.03,22.96]$} & {$[33.54,20.67]$} & {$[38.71,25.42]$} & {$[37.90,23.27]$} \\
\hline 0.30 & {$[22.44,15.06]$} & {$[22.44,12.86]$} & {$[25.09,17.14]$} & {$[24.95,14.68]$} \\
\hline 0.40 & {$[10.53,8.28]$} & {$[10.56,5.66]$} & {$[11.75,8.77]$} & {$[11.79,6.34]$} \\
\hline 0.50 & {$[5.54,5.91]$} & {$[5.19,2.55]$} & {$[5.97,6.13]$} & {$[5.62,2.90]$} \\
\hline 0.60 & {$[3.69,5.19]$} & {$[2.27,0.93]$} & {$[3.84,5.26]$} & {$[2.53,1.14]$} \\
\hline 0.70 & {$[3.14,5.04]$} & {$[0.91,0.37]$} & {$[3.17,5.04]$} & {$[0.97,0.41]$} \\
\hline 0.80 & {$[3.02,5.00]$} & {$[0.44,0.09]$} & {$[3.04,5.01]$} & {$[0.38,0.11]$} \\
\hline 0.90 & {$[3,5]$} & {$[0.05,0.01]$} & {$[3,5]$} & {$[0.09,0.04]$} \\
\hline
\end{tabular}

Table 3 The values of NARL and NSDRL under MDS sampling when $n_{N}=[3,5] ; m_{N}=[2,4]$; $\lambda_{N}=[0.28,0.32]$

\begin{tabular}{|c|c|c|c|c|}
\hline \multirow{4}{*}{$\begin{array}{l}k 1_{N} \\
k 2_{N} \\
\delta\end{array}$} & \multirow{2}{*}{\multicolumn{2}{|c|}{$\frac{r_{0}=300}{[2.97580,2.99004]}$}} & \multicolumn{2}{|l|}{$r_{0}=370$} \\
\hline & & & \multicolumn{2}{|l|}{$[3.08091,3.06453]$} \\
\hline & \multicolumn{2}{|l|}{$[2.50699,2.52689]$} & \multicolumn{2}{|l|}{$[2.55509,2.58679]$} \\
\hline & NARL & NSDRL & NARL & NSDRL \\
\hline 0.00 & {$[300.63,301.05]$} & {$[286.79,309.48]$} & {$[373.05,371.57]$} & {$[368.03,361.01]$} \\
\hline 0.05 & {$[262.81,242.24]$} & {$[256.01,235.82]$} & {$[317.99,295.27]$} & {$[310.47,291.59]$} \\
\hline 0.10 & {$[172.89,142.95]$} & {$[172.75,138.23]$} & {$[209.43,168.77]$} & {$[203.82,164.45]$} \\
\hline 0.15 & {$[110.49,82.26]$} & {$[107.78,79.02]$} & {$[129.05,95.61]$} & {$[127.76,91.5]$} \\
\hline 0.20 & {$[72.57,50.15]$} & {$[70.24,46.35]$} & {$[80.11,56.09]$} & {$[77.17,53.14]$} \\
\hline 0.25 & {$[47.97,31.92]$} & {$[45.53,28.95]$} & {$[53.11,35.69]$} & {$[51.64,32.28]$} \\
\hline 0.30 & {$[32.77,21.86]$} & {$[31.4,18.76]$} & {$[36.15,23.84]$} & {$[34.24,20.83]$} \\
\hline 0.40 & {$[16.27,11.49]$} & {$[15.36,8.51]$} & {$[18.48,12.48]$} & {$[17.33,9.41]$} \\
\hline 0.50 & {$[9.50,7.69]$} & {$[8.60,4.66]$} & {$[10.24,8.00]$} & {$[9.26,4.79]$} \\
\hline 0.60 & {$[6.06,5.96]$} & {$[5.01,2.25]$} & {$[6.39,6.13]$} & {$[5.46,2.47]$} \\
\hline 0.70 & {$[4.30,5.33]$} & {$[2.89,1.08]$} & {$[4.51,5.39]$} & {$[3.09,1.21]$} \\
\hline 0.80 & {$[3.49,5.10]$} & {$[1.58,0.48]$} & {$[3.57,5.12]$} & {$[1.65,0.57]$} \\
\hline 0.90 & {$[3.18,5.03]$} & {$[0.82,0.25]$} & {$[3.20,5.03]$} & {$[0.86,0.26]$} \\
\hline 1.00 & {$[3.05,5.01]$} & {$[0.4,0.1]$} & {$[3.06,5.01]$} & {$[0.43,0.11]$} \\
\hline
\end{tabular}

\section{Comparison Study of the Proposed Chart}

Literature on control charts show that a proposed chart is considered efficient if the ARL and the standard deviation of ARL values are smaller than the counterpart chart under the same parametric settings. Thus the proposed chart has been compared with the [60] chart which had been developed for the process monitoring under the neutrosophic EWMA statistics. Table 5 has been given for the comparison of the proposed MDS sampling with the [60] using the same parametric settings of $n=[3,5] ; \lambda=[0.08,0.12]$ for $\mathrm{ARL}_{0}=\{300$ and 370$\}$. From Table 5 it can be observed that the proposed chart has smaller values of ARL and SDARL for different values process shift. For instance, the small shift of 0.05 is detected in [210.81, 197.3] samples when $\mathrm{ARL}_{0}=\{300\}$ when the proposed chart is used while the same amount of shift is detected using [220.34, 202.5] samples when 
Table 4 The values of NARL and NSDRL under MDS sampling when $r_{0}=370$

\begin{tabular}{|c|c|c|c|c|}
\hline \multirow{4}{*}{$\begin{array}{l}k 1_{N} \\
k 2_{N} \\
\delta\end{array}$} & \multicolumn{2}{|c|}{$n_{N}=[5,7] ; m_{N}=[2,4]$} & \multicolumn{2}{|c|}{$n_{N}=[8,10] ; m_{N}=[2,4]$} \\
\hline & \multicolumn{2}{|c|}{$[3.05698,3.10119]$} & \multicolumn{2}{|c|}{$[2.73998,2.94916]$} \\
\hline & \multicolumn{2}{|l|}{$[2.4442,2.51186]$} & \multicolumn{2}{|c|}{$[2.47116,2.52955]$} \\
\hline & NARL & NSDRL & NARL & NSDRL \\
\hline 0.00 & {$[383.51,371.22]$} & {$[362.77,353.5]$} & {$[370.13,374.57]$} & {$[357.81,360.6]$} \\
\hline 0.05 & {$[213.25,208.01]$} & {$[208.08,205.91]$} & {$[169.01,175.02]$} & {$[166.96,169.67]$} \\
\hline 0.10 & {$[90.15,79.15]$} & {$[89.86,77.74]$} & {$[55.14,58.34]$} & {$[56.04,56.92]$} \\
\hline 0.15 & {$[38.54,35.46]$} & {$[40.67,34.12]$} & {$[18.94,23.28]$} & {$[22.61,23.03]$} \\
\hline 0.20 & {$[15.98,17.69]$} & {$[19.68,17.63]$} & {$[6.31,10.77]$} & {$[8.23,9.96]$} \\
\hline 0.25 & {$[6.67,9.64]$} & {$[8.63,8.93]$} & {$[3.34,6.51]$} & {$[2.16,4.22]$} \\
\hline 0.30 & {$[3.63,6.48]$} & {$[2.99,4.24]$} & {$[3.01,5.31]$} & {$[0.28,1.61]$} \\
\hline 0.40 & {$[3.00,5.08]$} & {$[0.05,0.65]$} & {$[3,5]$} & {$[0,0.1]$} \\
\hline 0.50 & {$[3,5]$} & {$[0,0.05]$} & {$[3,5]$} & {$[0,0]$} \\
\hline
\end{tabular}

Table 5 Comparison of the proposed chart with the existing chart

\begin{tabular}{|c|c|c|c|c|c|c|c|c|}
\hline \multirow{5}{*}{$\begin{array}{l}k 1_{N} \\
k 1_{N} \\
\delta\end{array}$} & \multicolumn{4}{|l|}{ Proposed } & \multicolumn{4}{|c|}{ Existing chart proposed by [60] } \\
\hline & \multicolumn{2}{|l|}{$r_{0}=300$} & \multicolumn{2}{|l|}{$r_{0}=370$} & \multicolumn{2}{|l|}{$r_{0}=300$} & \multicolumn{2}{|l|}{$r_{0}=370$} \\
\hline & \multicolumn{2}{|l|}{$[2.71534,3.33017]$} & \multicolumn{2}{|l|}{$[2.70613,2.98759]$} & \multicolumn{2}{|l|}{$k_{N}=[2.565,2.675]$} & \multicolumn{2}{|l|}{$k_{N}=[2.655,2.765]$} \\
\hline & \multicolumn{2}{|l|}{$[2.36204,2.42202]$} & \multicolumn{2}{|l|}{$[2.49637,2.52893]$} & & & & \\
\hline & NARL & NSDRL & NARL & NSDRL & NARL & NSDRL & NARL & NSDRL \\
\hline 0.00 & {$[302.88,299.88]$} & {$[290.18,293.27]$} & {$[378.95,382.23]$} & {$[364.49,375.83]$} & {$[306.19,301.49$} & {$[288.72,289.56]$} & {$[368.28,376.77]$} & {$[345.26,354.91]$} \\
\hline 0.05 & {$[210.81,197.3]$} & {$[203.91,190.27]$} & {$[254.12,235.41]$} & {$[251.11,233.44]$} & {$[220.34,202.5]$} & {$[206.84,195.15]$} & {$[270.32,248.92]$} & {$[257.15,238.27]$} \\
\hline 0.10 & {$[111.12,90.02]$} & {$[109.98,86.06]$} & {$[128.67,108.11]$} & {$[125.02,105.62]$} & {$[121.84,99.72$} & {$[109.75,93.06]$} & {$[141.33,117.16]$} & {$[130.78,106.3]$} \\
\hline 0.15 & {$[56.62,45.30]$} & {$[58.06,44.43]$} & {$[66.95,50.16]$} & {$[66.19,48.64]$} & {$[71.34,53.39]$} & {$[61.32,45.2]$} & {$[80.28,61.22]$} & {$[68.96,53.3]$} \\
\hline 0.20 & {$[29.90,24.31]$} & {$[32.85,23.77]$} & {$[35.71,27.13]$} & {$[39.41,26.94]$} & {$[45.6,33.38]$} & {$[36.07,25.93]$} & {$[50.59,36.58]$} & {$[39.41,28.44]$} \\
\hline 0.25 & {$[15.19,13.89]$} & {$[19.01,13.54]$} & {$[17.75,16.64]$} & {$[21.19,15.5]$} & {$[32.02,22.68]$} & {$[22.98,15.89]$} & {$[34.7,24.71]$} & {$[24.42,17.5]$} \\
\hline 0.30 & {$[7.54,8.69]$} & {$[10.0,7.66]$} & {$[8.94,9.40]$} & {$[11.8,8.24]$} & {$[24.18,16.77]$} & {$[15.29,10.67]$} & {$[25.78,18.28]$} & {$[16.59,11.86]$} \\
\hline 0.40 & {$[3.30,5.41]$} & {$[1.99,1.90]$} & {$[3.50,5.54]$} & {$[2.60,2.17]$} & {$[15.69,10.75]$} & {$[8.58,5.6]$} & {$[16.62,11.53]$} & {$[9.06,6.2]$} \\
\hline 0.50 & {$[3.01,5.02]$} & {$[0.30,0.32]$} & {$[3.01,5.03]$} & {$[0.21,0.46]$} & {$[11.67,8]$} & {$[5.47,3.69]$} & {$[12.24,8.19]$} & {$[5.83,3.66]$} \\
\hline 0.60 & {$[3,5]$} & {$[0,0.03]$} & {$[3,5]$} & {$[0,0.03]$} & {$[9.16,6.21]$} & {$[3.86,2.47]$} & {$[9.57,6.52]$} & {$[4,2.59]$} \\
\hline
\end{tabular}

the existing chart is used. Further a small shift of 0.50 is detected in [3.01, 5.02] samples when the existing chart is used when $\mathrm{ARL}_{0}=\{300\}$ while the same amount of shift is detected using [220.34, 202.5] samples when the existing chart is used. In addition when $\mathrm{ARL}_{0}=\{370\}$ the small shift of 0.05 is detected in [254.12, 235.41] samples when the proposed chart is used while the same amount of shift is detected using [270.32, 248.92] samples when the existing chart is used. Further a small shift of 0.50 is detected in [3.01, 5.03] samples when the existing chart is used while the same amount of shift is detected using [12.24, 8.19] samples when the existing chart is used. The same pattern can be seen for all values of shift. So it can be claimed that the proposed chart is comparatively efficient in the quick detection of smaller shifts in the production process.
A simulation study of the proposed chart has also been carried out to detect the working of the proposed methodology. Table 6 has been produced for the data from the NND in which neutrosophic mean values, $\bar{X}_{N} \in\left\{\bar{X}_{L}, \bar{X}_{U}\right\}$ and the neutrosophic EWMA, EWMA ${ }_{N}$ statistic have been calculated. The first 20 observations have been generated for the in-control process while the next 20 observations have been generated for the out-of-control process with a shift of $d=0.25$. Now we suppose that in our process the $n_{N} \in[5,7], \mathrm{ARL}_{0 N} \in[370,370]$ and $\lambda_{N} \in[0.08,0.12]$. From Table 4 the calculated NARL is $\mathrm{ARL}_{1 N} \in[6.67,9.64]$ which indicates that the process will be declared as out-ofcontrol in-between the seventh and tenth sample of the process having these parametric settings. The out-of-control 
Table 6 The simulated EWMA neutrosophic data

\begin{tabular}{lccccc}
\hline Sr.\# & $\bar{X}_{N}$ & EWMA $_{N}$ & Sr.\# & $\bar{X}_{N}$ & EWMA \\
\hline 1 & {$[74.00059,74.00044]$} & {$[74.00011,74.00011]$} & 21 & {$[74.00027,74.00113]$} & {$[74.0000,74.00001]$} \\
2 & {$[73.99905,73.99945]$} & {$[74.00003,74.00003]$} & 22 & {$[74.00008,73.99999]$} & {$[74.00001,74.00001]$} \\
3 & {$[74.00011,73.99964]$} & {$[74.00004,73.99999]$} & 23 & {$[74.00113,74.00023]$} & {$[74.0001,74.00004]$} \\
4 & {$[73.99962,73.99938]$} & {$[74.0000,73.99991]$} & 24 & {$[73.99956,74.00156]$} & {$[74.00005,74.00022]$} \\
5 & {$[73.9996,74.00042]$} & {$[73.99997,73.99997]$} & 25 & {$[73.9998,73.99943]$} & {$[74.00003,74.00012]$} \\
6 & {$[74.00078,74.0002]$} & {$[74.00003,74.0000]$} & 26 & {$[74.00006,74.0000]$} & {$[74.00004,74.00011]$} \\
7 & {$[73.99977,73.99828]$} & {$[74.00001,73.9998]$} & 27 & {$[74.00092,74.00062]$} & {$[74.00011,74.00017]$} \\
8 & {$[73.99927,73.99927]$} & {$[73.99995,73.99973]$} & 28 & {$[74.0002,73.99978]$} & {$[74.00011,74.00012]$} \\
9 & {$[74.00012,74.00026]$} & {$[73.99997,73.9998]$} & 29 & {$[74.00071,73.99972]$} & {$[74.00016,74.00008]$} \\
10 & {$[73.99957,74.0001]$} & {$[73.99994,73.99983]$} & 30 & {$[73.99969,74.00088]$} & {$[74.00012,74.00017]$} \\
11 & {$[74.00017,74.00127]$} & {$[73.99995,74.00001]$} & 31 & {$[74.00029,74.00046]$} & {$[74.00014,74.00021]$} \\
12 & {$[74.00013,73.99922]$} & {$[73.99997,73.99991]$} & 32 & {$[74.00032,73.99918]$} & {$[74.00015,74.00008]$} \\
13 & {$[73.99998,73.99927]$} & {$[73.99997,73.99983]$} & 33 & {$[74.00057,74.00092]$} & {$[74.00019,74.00018]$} \\
14 & {$[73.99949,73.99962]$} & {$[73.99993,73.99981]$} & 34 & {$[74.00007,74.00082]$} & {$[74.00018,74.00026]$} \\
15 & {$[73.99959,73.99956]$} & {$[73.9999,73.99978]$} & 35 & {$[74.00106,74.0018]$} & {$[74.00025,74.00045]$} \\
16 & {$[73.99991,73.99899]$} & {$[73.9999,73.99968]$} & 36 & {$[74.0005,74.00056]$} & {$[74.00027,74.00046]$} \\
17 & {$[73.99968,73.99988]$} & {$[73.99989,73.99971]$} & 37 & {$[74.00003,73.99957]$} & {$[74.00025,74.00035]$} \\
18 & {$[74.00005,74.00015]$} & {$[73.9999,73.99976]$} & 38 & {$[73.99921,74.00022]$} & {$[74.00016,74.00034]$} \\
19 & {$[73.99986,74.00038]$} & {$[73.9999,73.99984]$} & 39 & {$[74.00009,74.00027]$} & {$[74.00016,74.00033]$} \\
20 & {$[74.0009,74.00005]$} & {$[73.99998,73.99986]$} & 40 & {$[74.00007,73.99982]$} & {$[74.00015,74.00027]$} \\
\hline & & & & &
\end{tabular}

Fig. 1 The proposed chart for simulated data (left) and existing chart proposed by [60] for the simulated data (right)

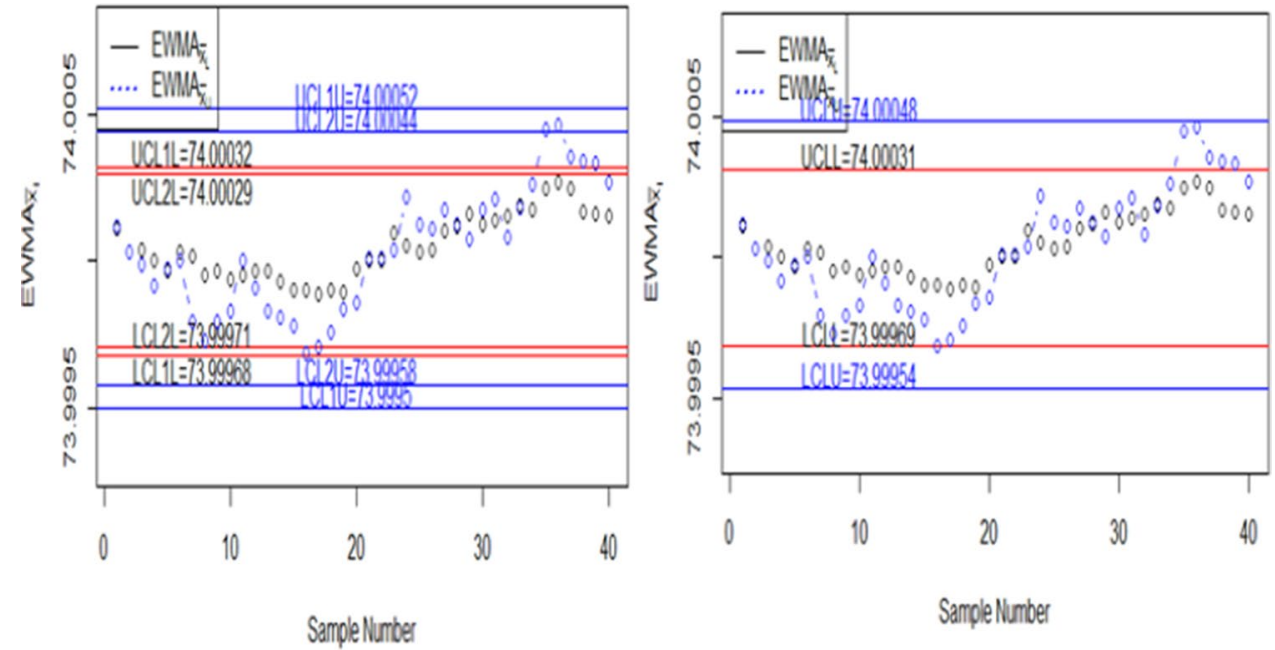

process after the seventh and tenth has also been shown in Fig. 1 of the proposed chart simulation study with the chart coefficients $k 1=[2.70613,2.98759]$ and $k 2=[2.49637,2.53893]$ and the two pairs of control limits as $\operatorname{LCL1}_{N}=[73.9995,73.99968]$, $\operatorname{LCL}_{N}=[73.99958,73.99971], \mathrm{UCL}_{N}=[74.00029,74.00044]$ and $\mathrm{UCL}_{N}=[74.00032,74.00052]$ For the purpose of comparative study, Fig. 1 has been generated with the same process settings having applied on the existing chart presented by
[60] which shows that the process is in-control and unable to detect this shift. On observing Fig. 1 it can be concluded that the proposed chart is comparatively efficient in quick detection of the out-of-control process for any small shift being faced by the production process. To study robustness, we have conducted a comparative study with existing studies and also various parametric combinations. From simulation and comparative study the proposed methodology shows the 
Table 7 Real example $n_{N}=[5$, 7] and $\lambda_{N}=[0.08,0.12]$

\begin{tabular}{lllllllll}
\hline Months & Saturday & Sunday & Monday & Tuesday & Wednesday & Thursday & Friday & EWMA $_{\mathrm{N}}$ \\
\hline January & 426 & 601 & 596 & 586 & 574 & 583 & 407 & {$[490.94,491.68]$} \\
February & 487 & 812 & 525 & 476 & 421 & 498 & 413 & {$[495.20,494.94]$} \\
March & 406 & 789 & 551 & 427 & 412 & 498 & 398 & {$[496.94,495.22]$} \\
April & 448 & 614 & 458 & 407 & 491 & 486 & 407 & {$[495.87,492.56]$} \\
May & 423 & 611 & 518 & 457 & 427 & 482 & 412 & {$[495.18,490.53]$} \\
June & 530 & 590 & 563 & 475 & 479 & 511 & 372 & {$[497.76,492.01]$} \\
July & 493 & 623 & 511 & 587 & 587 & 528 & 396 & {$[502.75,496.83]$} \\
August & 453 & 652 & 579 & 578 & 552 & 503 & 427 & {$[507.56,501.39]$} \\
September & 491 & 546 & 503 & 498 & 488 & 517 & 410 & {$[507.37,500.42]$} \\
October & 378 & 412 & 422 & 413 & 382 & 456 & 373 & {$[498.89,488.99]$} \\
November & 394 & 533 & 449 & 380 & 393 & 405 & 394 & {$[493.36,480.84]$} \\
December & 402 & 576 & 517 & 397 & 388 & 419 & 307 & {$[490.37,474.67]$} \\
\hline
\end{tabular}

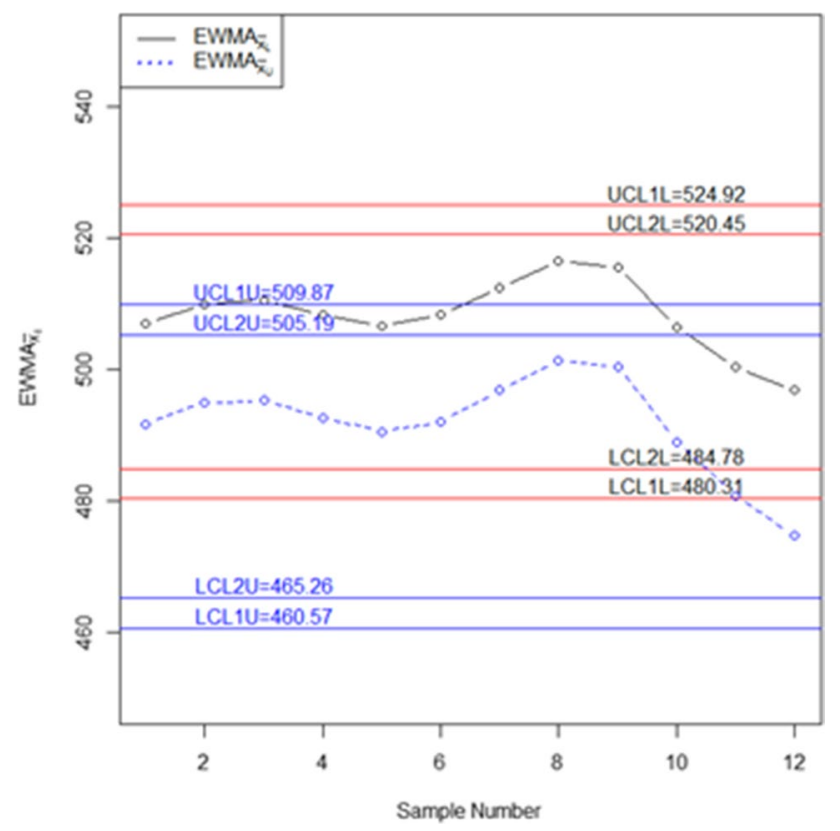

Fig. 2 The proposed chart for monitoring road accidents

robustness and validation in the field of statistical process control.

\section{Application of the Proposed Chart in Monitoring Road Accidents and Injuries}

\subsection{Application 1 (Monitoring Road Accidents)}

In this section the practical application of the proposed chart has been given. The proposed methodology has been developed for the quick monitoring of the neutrosophic EWMA statistic using the MDS sampling. As stated above the proposed chart is quite effective, efficient

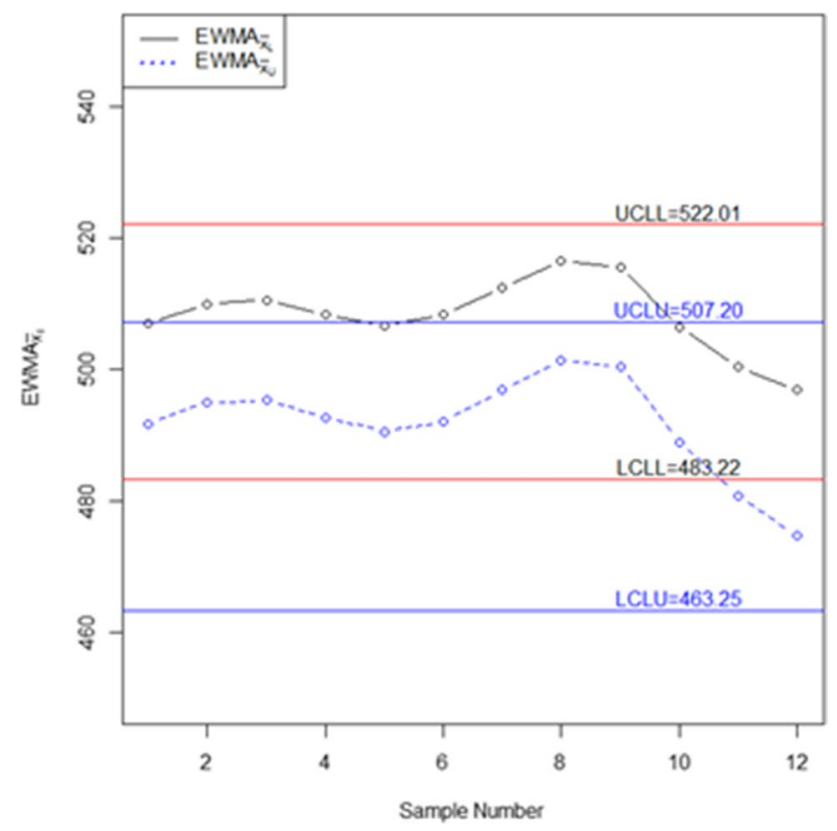

Fig. 3 The existing chart for monitoring road accidents by [60]

and flexible to monitor the process for the neutrosophic statistics. Therefore the practical application has been given using the data related to road accident from Saudi Arabia for daily basis of the year. The aim of this application is to monitor the road accidents on the various days of the week. For ready reference data is shown in Table 7 along with the neutrosophic EWMA statistics of the reference data are calculated. The calculated limits are given using $n_{N}=[5,7], \mu_{N}=[502.62,485.23]$ and $\sigma_{N}=[79.92,83.24]$. The control chart coefficients are calculated as $k 1=[3.05698,3.10119]$ and $k 2=[2.4442,2.51186]$. Thus the two pairs of 
Table 8 Real example $n_{N}=$ $[3,5]$ and $\lambda_{N}=[0.08,0.12]$

\begin{tabular}{lllllll}
\hline Months & Age & & & & & \\
\cline { 2 - 5 } & Less than 18 & $18-30$ & $30-40$ & $40-50$ & More than 50 & \\
\hline January & 14 & 59 & 62 & 49 & 27 & {$[54.56,48.55]$} \\
February & 11 & 61 & 54 & 39 & 41 & {$[53.55,47.67]$} \\
March & 21 & 92 & 71 & 41 & 48 & {$[54.18,48.50]$} \\
April & 16 & 79 & 61 & 36 & 36 & {$[54.00,48.15]$} \\
May & 12 & 74 & 61 & 29 & 23 & {$[53.6,47.15]$} \\
June & 18 & 86 & 75 & 33 & 29 & {$[54.09,47.28]$} \\
July & 15 & 76 & 61 & 29 & 38 & {$[53.81,46.86]$} \\
August & 22 & 89 & 88 & 55 & 44 & {$[54.81,48.39]$} \\
September & 25 & 103 & 92 & 62 & 55 & {$[56.30,50.67]$} \\
October & 15 & 89 & 74 & 48 & 34 & {$[55.91,50.47]$} \\
November & 17 & 74 & 55 & 54 & 39 & {$[56.02,50.51]$} \\
December & 15 & 96 & 61 & 44 & 38 & \\
\hline
\end{tabular}

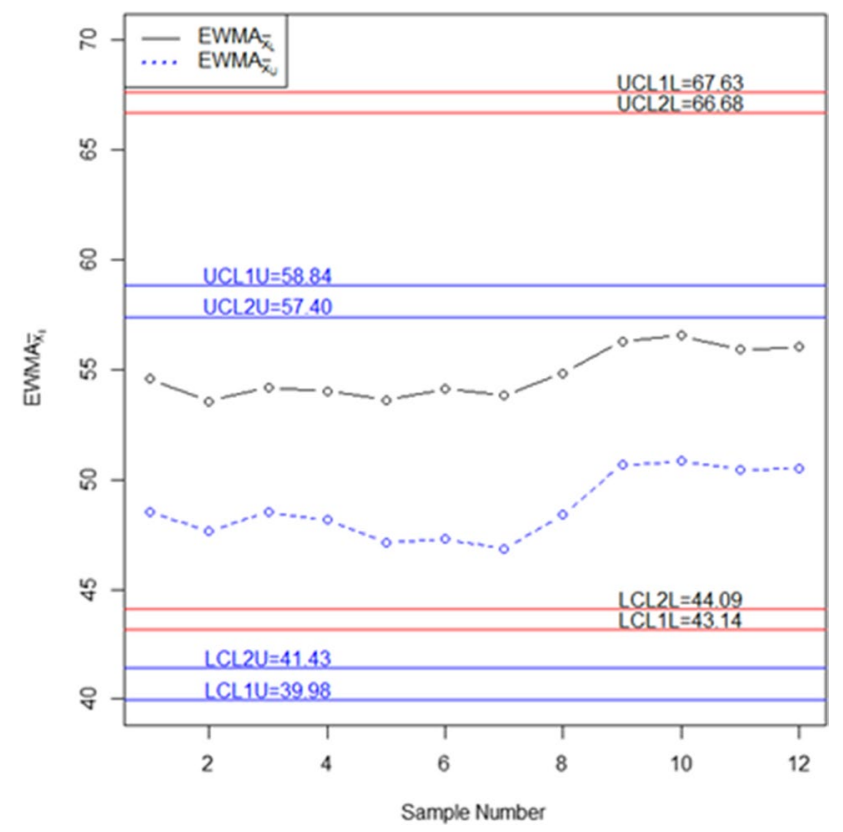

Fig. 4 The proposed chart for monitoring road injuries

limits are calculated as $\mathrm{LCL}_{N}=[477.97,480.31]$, $\operatorname{LCL}_{N}=[482.65,484.64], \quad \mathrm{UCL} 2_{N}=[520.45,522.58]$ and $\mathrm{UCL}_{N}=[524.92 .88,527.27]$. Figure 2 has been given for the proposed chart methodology and Fig. 3 has been given for the existing chart proposed by [60]. It can be observed that the proposed chart provide the narrow limits as compared to the existing chart.

\subsection{Application 2 (Monitoring Road Injuries)}

Furthermore, one more real-life application for the developed control chart is illustrated in this sub-section. The accident data of indeterminate nature different

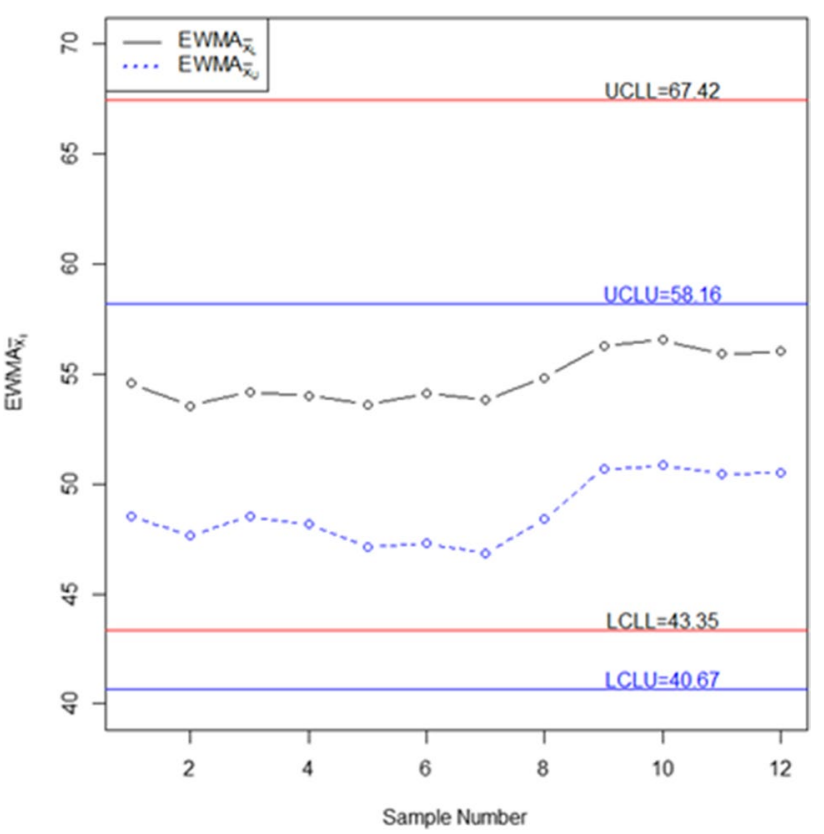

Fig. 5 The existing chart for monitoring road injuries by [60]

age groups of people in various months of the year is described in Table 8. The coefficients of control limits are $k 1=[2.70613,2.98759]$ and $k 2=[2.49637,2.52893]$ using $n_{N}=[3,5], \mu_{N}=[55.39,49.42]$ and $\sigma_{N}=[38.39,27.94]$. The following two pairs of control limits are calculated $\operatorname{LCL}_{N}=[39.98,43.14], \mathrm{LCL} 2_{N}=[41.43,44.09]$, $\mathrm{UCL} 2_{N}=[57.40,66.68]$ and $\mathrm{UCL} 1_{N}=[58.84,67.63]$ and given in Fig. 4. Figure 4 has been given for the proposed chart methodology and Fig. 5 has been given for the existing chart proposed by [60]. Here again, it can be observed 
that the proposed chart provides narrow limits as compared to the existing chart.

\section{Concluding Remarks}

In this paper, neutrosophic multiple dependent state sampling control chart for the neutrosophic EWMA statistic has been presented. The coefficients of the proposed chart have been determined using the neutrosophic statistical interval method for different process settings. The average run lengths and the standard deviation of the average run lengths have been determined using the Monte Carlo simulation. The comparison of the proposed chart with the existing chart has been discussed. It has been concluded that the proposed chart is the comparatively efficient chart for monitoring the vague, incomplete, unclear and non-crispy quality characteristics. The limitation of our study is the production process should follow the normal distribution. The proposed new X-bar control chart for multiple dependent state sampling using neutrosophic exponentially weighted moving average statistics could be applied to chemical, packing and electronic industries. Future study is suggested for the non-normal processes on chemical, packing and electronic industries employed using the proposed methodology.

Acknowledgements The authors are deeply thankful to the editor and reviewers for their valuable suggestions to improve the quality and presentation of the paper.

Author Contributions NK, LA, GSR, MA and AHAM wrote the paper.

Funding None.

Availability of Data and Materials The data is given in the paper.

\section{Declarations}

\section{Conflict of Interest None.}

Ethics Approval and Consent to Participate Not applicable.

Consent for Publication Not applicable.

Open Access This article is licensed under a Creative Commons Attribution 4.0 International License, which permits use, sharing, adaptation, distribution and reproduction in any medium or format, as long as you give appropriate credit to the original author(s) and the source, provide a link to the Creative Commons licence, and indicate if changes were made. The images or other third party material in this article are included in the article's Creative Commons licence, unless indicated otherwise in a credit line to the material. If material is not included in the article's Creative Commons licence and your intended use is not permitted by statutory regulation or exceeds the permitted use, you will need to obtain permission directly from the copyright holder. To view a copy of this licence, visit http://creativecommons.org/licenses/by/4.0/.

\section{References}

1. Aslam, M., et al.: Monitoring circuit boards products in the presence of indeterminacy. Measurement 168, 108404 (2021)

2. Saghir, A., et al.: Monitoring process variation using modified EWMA. Qual. Reliab. Eng. Int. 36(1), 328-339 (2020)

3. Ahmad, L., Aslam, M., Jun, C.-H.: Coal quality monitoring with improved control charts. Eur. J. Sci. Res. 125(2), 427-434 (2014)

4. Dull, R.B., Tegarden, D.P.: Using control charts to monitor financial reporting of public companies. Int. J. Account. Inf. Syst. 5(2), 109-127 (2004)

5. Woodall, W.H.: The use of control charts in health-care and public-health surveillance. Qual. Control Appl. Stat. 52(3), 253-256 (2007)

6. Hwang, S.-L., et al.: Application control chart concepts of designing a pre-alarm system in the nuclear power plant control room. Nucl. Eng. Des. 238(12), 3522-3527 (2008)

7. Huang, F.-H., et al.: Evaluation and comparison of alarm reset modes in advanced control room of nuclear power plants. Saf. Sci. 44(10), 935-946 (2006)

8. Mahanti, R., Evans, J.R.: Critical success factors for implementing statistical process control in the software industry. Benchmark. Int. J. 19(3), 374-394 (2012)

9. Tunca, M.Z., Sutcu, A.: Use of statistical process control charts to assess web quality: an investigation of online furniture stores. Int. J. Electron. Bus. 4(1), 40-55 (2006)

10. Wang, Z. and R. Liang. Discuss on applying SPC to quality management in university education. In: The 9th International Conference for Young Computer Scientists. IEEE (2008).

11. Masson, P.: Quality control techniques for routine analysis with liquid chromatography in laboratories. J. Chromatogr. A 1158(12), 168-173 (2007)

12. Carson, P.K., Yeh, A.B.: Exponentially weighted moving average (EWMA) control charts for monitoring an analytical process. Ind. Eng. Chem. Res. 47(2), 405-411 (2008)

13. Wortham, A.W., Baker, R.C.: Multiple deferred state sampling inspection. Int. J. Prod. Res. 14(6), 719-731 (1976)

14. Aslam, M., Khan, N., Jun, C.-H.: A multiple dependent state control chart based on double control limit. Res. J. Appl. Sci. Eng. Technol. 7(21), 4490-4493 (2014)

15. Aslam, M., et al.: A control chart for an exponential distribution using multiple dependent state sampling. Qual. Quant. 49(2), 455-462 (2015)

16. Wu, C.-W., Liu, S.-W., Lee, A.H.: Design and construction of a variables multiple dependent state sampling plan based on process yield. Eur. J. Ind. Eng. 9(6), 819-838 (2015)

17. Balamurali, S., Jeyadurga, P., Usha, M.: Designing of Bayesian multiple deferred state sampling plan based on gamma-Poisson distribution. Am. J. Math. Manag. Sci. 35(1), 77-90 (2016)

18. Yan, A., Liu, S., Dong, X.: Designing a multiple dependent state sampling plan based on the coefficient of variation. Springerplus 5(1), 1447 (2016)

19. Aldosari, M.S., Aslam, M., Jun, C.-H.: A new attribute control chart using multiple dependent state repetitive sampling. IEEE Access (2017). https://doi.org/10.1177/0142331214549094

20. Zhou, W., et al.: A joint-adaptive np control chart with multiple dependent state sampling scheme. Commun. Stat. 46(14), 69676979 (2017)

21. Gülbay, M., Kahraman, C.: An alternative approach to fuzzy control charts: direct fuzzy approach. Inf. Sci. 177(6), 1463-1480 (2007)

22. Zadeh, L.A.: Fuzzy sets. Inform. Control. 8, 338-353 (1965) 
23. Raz, T., Wang, J.-H.: Probabilistic and membership approaches in the construction of control charts for linguistic data. Prod. Plan. Control 1(3), 147-157 (1990)

24. Gülbay, M., Kahraman, C.: Development of fuzzy process control charts and fuzzy unnatural pattern analyses. Comput. Stat. Data Anal. 51(1), 434-451 (2006)

25. Zarandi, M.F., Alaeddini, A., Turksen, I.: A hybrid fuzzy adaptive sampling-run rules for Shewhart control charts. Inf. Sci. 178(4), 1152-1170 (2008)

26. Panthong, C., Pongpullponsak, A.: Non-normality and the fuzzy theory for variable parameters control charts. Thai J. Math. 14(1), 203-213 (2016)

27. Afshari, R., Sadeghpour Gildeh, B.: Designing a multiple deferred state attribute sampling plan in a fuzzy environment. Am. J. Math. Manag. Sci. 36(4), 328-345 (2017)

28. Fernández, M.N.P. Fuzzy theory and quality control charts. In: 2017 IEEE International Conference on Fuzzy Systems (FUZZIEEE) (2017)

29. Smarandache, F.: Introduction to neutrosophic statistics: Infinite study. Sitech \& Education Publishing (2014)

30. Chen, J., Ye, J., Du, S.: Scale effect and anisotropy analyzed for neutrosophic numbers of rock joint roughness coefficient based on neutrosophic statistics. Symmetry 9(10), 208 (2017)

31. Aslam, M.: A new sampling plan using neutrosophic process loss consideration. Symmetry 10(5), 132 (2018)

32. Aslam, M., AL-Marshadi, M.: Design of sampling plan using regression estimator under indeterminacy. Symmetry $\mathbf{1 0}(12), 754$ (2018)

33. Aslam, M., Khan, N., Albassam, M.: Control chart for failurecensored reliability tests under uncertainty environment. Symmetry 10(12), 690 (2018)

34. Aslam, M., Khan, N., Khan, M.Z.: Monitoring the variability in the process using neutrosophic statistical interval method. Symmetry 10(11), 562 (2018)

35. Aslam, M., Bantan, R.A., Khan, N.: Design of a control chart for gamma distributed variables under the indeterminate environment. IEEE Access 7, 8858-8864 (2019)

36. Khan, Z., et al.: Design of S-control chart for neutrosophic data: an application to manufacturing industry. J. Intell. Fuzzy Syst. 38(4), 4743-4751 (2020)

37. Jana, C., Pal, M.: A robust single-valued neutrosophic soft aggregation operators in multi-criteria decision making. Symmetry 11(1), 110 (2019)

38. Jana, C., et al.: Trapezoidal neutrosophic aggregation operators and their application to the multi-attribute decision-making process. Sci. Iran. 27(3), 1655-1673 (2020)

39. Jana, C., Muhiuddin, G., Pal, M.: Multi-criteria decision making approach based on SVTrN Dombi aggregation functions. Artif. Intell. Rev. 54(5), 3685-3723 (2021)

40. Jana, C., Pal, M.: Multi-criteria decision making process based on some single-valued neutrosophic Dombi power aggregation operators. Soft. Comput. 25(7), 5055-5072 (2021)

41. Jana, C., Muhiuddin, G., Pal, M.: Multiple-attribute decision making problems based on SVTNH methods. J. Ambient. Intell. Humaniz. Comput. 11(9), 3717-3733 (2020)

42. Aslam, M., Arif, O.H.: Testing of grouped product for the weibull distribution using neutrosophic statistics. Symmetry 10(9), 403 (2018)

43. Peng, X., Dai, J.: A bibliometric analysis of neutrosophic set: two decades review from 1998 to 2017. Artif. Intell. Rev. 2018, 1-57 (2018)
44. Peng, X., Dai, J.: Approaches to single-valued neutrosophic MADM based on MABAC, TOPSIS and new similarity measure with score function. Neural Comput. Appl. 29(10), 939-954 (2018)

45. Abdel-Basset, M., Atef, A., Smarandache, F.: A hybrid neutrosophic multiple criteria group decision making approach for project selection. Cogn. Syst. Res. 57, 216-227 (2019)

46. Aslam, M.: A new failure-censored reliability test using neutrosophic statistical interval method. Int. J. Fuzzy Syst. 21(4), 1214-1220 (2019)

47. Aslam, M.: A variable acceptance sampling plan under neutrosophic statistical interval method. Symmetry 11(1), 114 (2019)

48. Aslam, M., Bantan, R.A., Khan, N.: Design of a new attribute control chart under neutrosophic statistics. Int. J. Fuzzy Syst. 21(2), 433-440 (2019)

49. Aslam, M., Raza, M.A.: Design of new sampling plans for multiple manufacturing lines under uncertainty. Int. J. Fuzzy Syst. 21(3), 978-992 (2019)

50. Islam, S., Deb, S.C.: Neutrosophic goal programming approach to a green supplier selection model with quantity discount. Neutrosophic Sets Syst. 30, 1 (2019)

51. Jansi, R., Mohana, K., Smarandache, F.: Correlation measure for Pythagorean neutrosophic sets with $\mathrm{T}$ and $\mathrm{F}$ as dependent neutrosophic components. Neutrosophic Sets Syst. 30, 11 (2019)

52. Kashif, M., et al.: Decomposition of matrix under neutrosophic environment. Neutrosophic Sets Syst. 30, 1 (2019)

53. Muralikrishna, P., Kumar, D.S.: Neutrosophic approach on normed linear space. Neutrosophic Sets Syst. 30, 22 (2019)

54. Villamar, C.M., et al.: Analysis of technological innovation contribution to gross domestic product based on neutrosophic cognitive maps and neutrosophic numbers. Neutrosophic Sets Syst. 30, 3 (2019)

55. Zhang, X., et al.: Singular neutrosophic extended triplet groups and generalized groups. Cogn. Syst. Res. 57, 32-40 (2019)

56. Aslam, M.: (2020) Analysing gray cast iron data using a new Shapiro-Wilks test for normality under indeterminacy. Int. J. Cast Metals Res. 34, 1-5 (2020)

57. Aslam, M., Albassam, M.: Presenting post hoc multiple comparison tests under neutrosophic statistics. J. King Saud Univ. Sci. 32, 2728-2732 (2020)

58. Saad, M., et al.: Computing shortest path in a single valued neutrosophic hesitant fuzzy network. Nucleus 56(3), 123-130 (2020)

59. Montgomery, C.D.: Introduction to statistical quality control, 6th edn. John Wiley \& Sons Inc, New York (2009)

60. Aslam, M., AL-Marshadi, A.H., Khan, N.: A New X-Bar Control chart for using neutrosophic exponentially weighted moving average. Mathematics 7(10), 957 (2019)

61. Şentürk, S., et al.: Fuzzy exponentially weighted moving average control chart for univariate data with a real case application. Appl. Soft Comput. 22, 1-10 (2014)

62. Montgomery, D.C.: Introduction to statistical quality control. John Wiley \& Sons, New York (2007)

Publisher's Note Springer Nature remains neutral with regard to jurisdictional claims in published maps and institutional affiliations. 